\title{
Paternal contribution to birth weight
}

\author{
P Magnus, H K Gjessing, A Skrondal, R Skjærven
}

\begin{abstract}
Study objective-Understanding causes of variation in birth weight has been limited by lack of sufficient sets of data that include paternal birth weight. The objective was to estimate risks of low birth weight dependent on parental birth weights and to estimate father-motheroffspring correlations for birth weight to explain the variability in birth weight in terms of effects of genes and environmental factors.
\end{abstract}

Design-A family design, using trios of father-mother-firstborn child.

Setting-The complete birth population in Norway 1967-98.

Participants-67 795 families.

Main results-The birth weight correlations were 0.226 for mother-child and 0.126 for father-child. The spousal correlation was low, 0.020 . The relative risk of low birth weight in the first born child was 8.2 if both parents were low birth weight themselves, with both parents being above $4 \mathrm{~kg}$ as the reference. The estimate of heritability is about 0.25 for birth weight, under the assumption that cultural transmission on the paternal side has no effect on offspring prenatal growth.

Conclusions-Paternal birth weight is a significant and independent predictor of low birth weight in offspring. The estimate of the heritability of birth weight in this study is lower than previously estimated from data within one generation in the Norwegian population.

(F Epidemiol Community Health 2001;55:873-877)

Population Healt

Sciences, Nationa

Institute of Public

Health, Oslo, Norway

$P$ Magnus

A Skrondal

Section for Medical Statistics, University of

Bergen, Bergen,

Norway

H K Gjessing

R Skjærven

Medical Birth Registry of Norway, University of Bergen, Bergen,

Norway

R Skjærven

Correspondence to: Dr Magnus, Section of Epidemiology, Department of Population Health Sciences, National Institute of Public Health, PO Box 4404 Nydalen, 0403 Oslo, Norway

(per.magnus@folkehelsa.no)

Accepted for publication 6 June 2001 may serve clinical purposes. The second aim is to understand the major causes of variability in prenatal growth, by analysing the intergenerational correlations in terms of genetic and environmental factors.

\section{Methods}

Each year, about 60000 births occur in Norway. The Medical Birth Registry of Norway comprises all births that have taken place since $1967 .{ }^{7}$ Through the 11 digit personal numbers, 67795 mother-father-child trios with complete data on birth weight were identified where all family members were born in the period 1967 to 1998 . To avoid major influences on birth weight associated with plurality, parity and early death, and to be able to compare the results with earlier findings, ${ }^{2}$ we included only singleton births for both generations and included only firstborn children who had survived the four first weeks of life. Birth weight is recorded immediately after birth to the nearest dekagram above the measured value. Low birth weight is defined as a weight below 2500 grams.

Relative risks of low birth weight were estimated from contingency tables based on parental birth weights. The phenotypic correlations were estimated as Pearson productmoment correlation coefficients. As systematic differences between males and females in mean values and standard deviations (table 1) for birth weight were found, $\mathrm{z}$ scores were created for sons, daughters, mothers and fathers before estimating gender adjusted correlation coefficients between relatives.

Population attributable risks were defined as $(\mathrm{p}-\mathrm{q}) / \mathrm{p}$, were $\mathrm{p}$ is the probability that a child randomly selected from our sample had low birth weight, and q the corresponding probability under a modified covariate distribution. The modified covariate distribution was constructed by shifting parents in the two lowest weight categories to the mid category $(3000 \mathrm{~g}$ $-3499 \mathrm{~g}$ ). We first estimated $\mathrm{p}$ by the average of the predicted probabilities from a logistic regression based on our sample, discarding interactions as these were non-significant. The q value was estimated by the average of the predicted probabilities under the modified covariate distribution, using the parameters estimated from our sample.

For the analysis of genetic and environmental effects, a path diagram (fig 1) was set up to represent latent (circles) and observed (squares) variables. Fw, Mw and $\mathrm{Cw}$ means the observed paternal, maternal and child birth weights, while $F_{G}, M_{G}$ and $C_{G}$ represent the unobserved genotypic values ${ }^{8}$ that influence birth weight, with an effect $h$. We assume that the genotypic value is a sum of effects of many genes, each with a small effect, without 


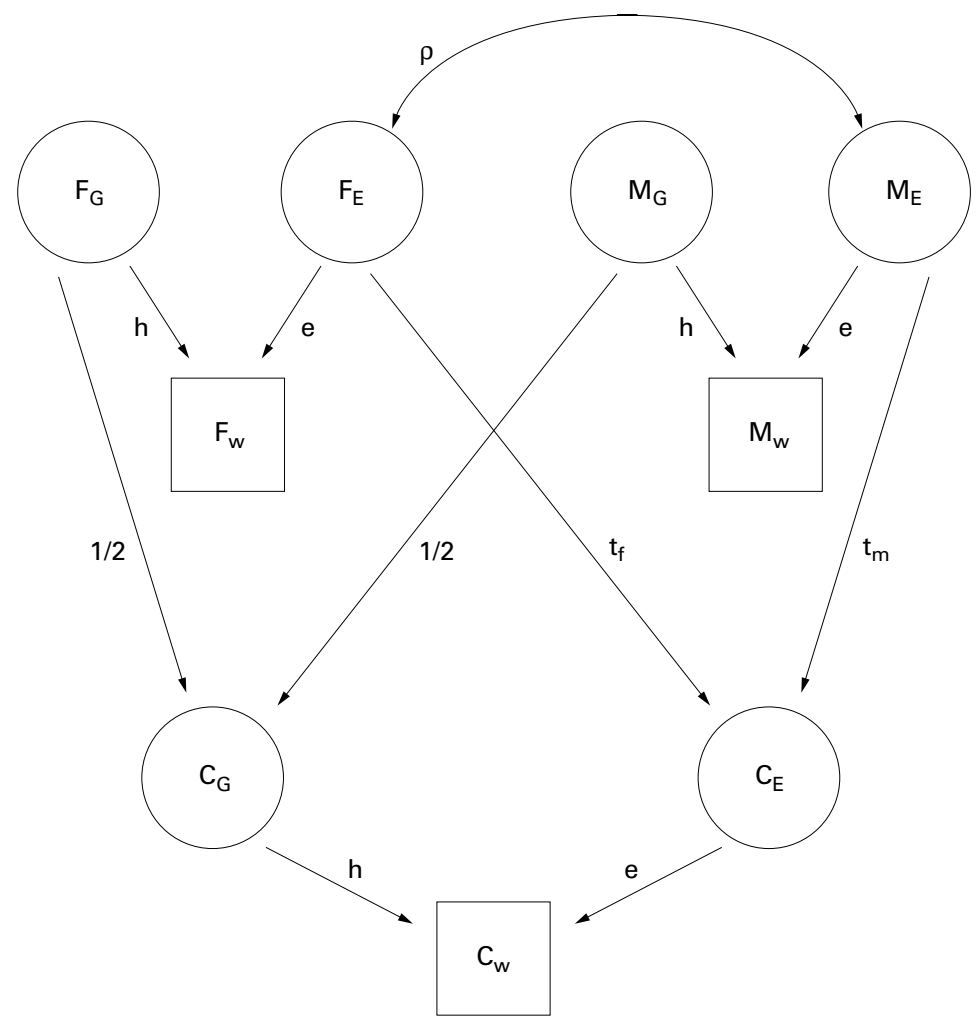

Figure 1 Path analytical diagram of paternal (Fw), maternal (Mw) and child (Cw) birth weight (shown as squares) as determined by the unobserved genotypic $(G)$ and environmental (E) values (shown as circles).

intralocus (dominance) or interlocus (epistasis) interactions. Furthermore, we designate by $\mathrm{F}_{\mathrm{E}}, \mathrm{M}_{\mathrm{E}}$ and $\mathrm{C}_{\mathrm{E}}$ the unobserved environmental values that influence birth weight, with an effect e, and hypothesise that there are effects $\left(t_{f}\right.$ and $t_{m}$ ) from the environments that influenced the parental birth weights to the environment that influence the child's birth weight. Residuals for the unobserved variables are not drawn in the figure, and the variables are assumed to have zero as mean value and one as variance. Finally, a correlation $(\rho)$ between the parental environments is assumed, representing a certain degree of social homogamy rather than assortative mating for the phenotype in question. The unknown effects

Table 1 Distributions of birth weight, proportion of low birth weight births, age at childbirth and year of birth for subjects belonging to 67795 mother-father-child trios (35 048 with sons and 32747 with daughters) as registered in the Norwegian Medical Birth Registry 1967-98

\begin{tabular}{|c|c|c|c|c|}
\hline & Father & Mother & Son & Daughter \\
\hline \multicolumn{5}{|l|}{ Birth weight (g) } \\
\hline Mean value & 3581 & 3429 & 3555 & 3452 \\
\hline Standard deviation & 531 & 504 & 580 & 540 \\
\hline Per cent of births with low birth weight & 2.6 & 3.3 & 4.0 & 3.9 \\
\hline \multicolumn{5}{|l|}{ Age at childbirth (y) } \\
\hline Mean value & 24.7 & 23.1 & & \\
\hline Standard deviation & 3.0 & 3.1 & & \\
\hline \multicolumn{5}{|l|}{ Year of birth, number of subjects } \\
\hline $1967-69$ & 34237 & 18509 & & \\
\hline $1970-72$ & 21864 & 25095 & & \\
\hline $1973-75$ & 9110 & 16315 & & \\
\hline $1976-78$ & 2294 & 6663 & & \\
\hline $1979-81$ & 286 & 1195 & & \\
\hline $1982-84$ & 4 & 18 & 3 & 2 \\
\hline $1985-87$ & & & 369 & 295 \\
\hline $1988-90$ & & & 2666 & 2517 \\
\hline $1991-93$ & & & 7395 & 6775 \\
\hline 1994-96 & & & 13671 & 12815 \\
\hline 1997-98 & & & 10944 & 10343 \\
\hline
\end{tabular}

( $h, e, t_{f}, t_{m}$ and $\rho$ ) can be related to the observed phenotypic correlations by setting up structural equations, using the principles of path analysis. ${ }^{8}$ As the full set of equations is underdetermined, we explored two models with different restrictions on the parameters, see table 4. The first model assumes no cultural transmission on the father's side $\left(t_{\mathrm{f}}=0\right)$. In addition, we use the restriction $h^{2}+e^{2}=1$. The heritability $h^{2}$ is then the proportion of the total variance in birth weight that is explained by genes. The remaining variation $\mathrm{e}^{2}$ is the effect of environmental conditions (for example, $\mathrm{C}_{\mathrm{E}}$ for the child) on birth weight. The variation in environmental conditions $\left(\mathrm{C}_{\mathrm{E}}\right)$ is again decomposed into $\mathrm{t}_{\mathrm{m}}^{2}$, which is the amount transmitted across generations, and $1-\mathrm{t}_{\mathrm{m}}{ }^{2}$, which corresponds to other unspecified environmental effects (not drawn in picture). The second model assumes no genetic effect $(h=0)$. In addition, we use the restriction $\mathrm{t}_{\mathrm{f}}^{2}+\mathrm{t}_{\mathrm{m}}{ }^{2}=1$. Under this model, $\mathrm{C}_{\mathrm{E}}$ only represents the environmental conditions transmitted across generations, decomposed into $\mathrm{t}_{\mathrm{m}}{ }^{2}$ from the mother and $\mathrm{t}_{\mathrm{f}}{ }^{2}$ from the father. Accordingly, $\mathrm{e}^{2}$ measures how much of the birth weight variation that is determined by transmitted environmental conditions, and the residual $1-\mathrm{e}^{2}$ is attributable to unspecified environmental effects (not drawn in picture).

Subject to these restrictions, the set of equations for the first model can be solved explicitly (by hand) to yield the estimates of $h, e, t_{m}$ and $\rho$ (as functions of the observed correlations). By resampling from the trivariate birth weight distribution and recomputing the estimates for each new sample we then obtain the 95\% bootstrap confidence intervals for these parameters. ${ }^{9}$ The same procedure was followed for the second model, estimating the parameters e, $t_{m}, t_{f}$ and $\rho$. The bootstrapping was performed in S-PLUS 2000 for Windows. ${ }^{10}$

\section{Results}

The proportion of low birth weight fathers (2.6 $\%)$ was lower than the corresponding proportion for mothers $(3.3 \%)$, while there was no difference in the risk of low birth weight for sons and daughters (table 1). The difference in mean birth weight between fathers and mothers $(152 \mathrm{~g})$ was larger than the difference between male and female offspring (103 g). The variance in birth weight was larger for fathers compared with mothers and larger for sons than for daughters.

The mother-father correlation in birth weight is low (table 2). The mother-child correlations are larger than the father-child correlations (table 2). There is a slight tendency that parent-daughter correlations are larger than parent-son correlations. The gender standardised ( $\mathrm{z}$ scores) father-child and mother-child correlations $(0.130$ and 0.226 , respectively) were almost identical to the unadjusted coefficients presented in table 2 .

Figure 2 shows the almost linear increase in offspring birth weight as paternal birth weight increases, within categories of maternal birth weight. The figure omits families where the paternal birth weight is below 2500 grams. It should be noted that the lines are almost 
Table 2 Unadjusted correlation coefficients for birth weight in pairs of relatives as registered in the Norwegian Birth Registry 1967-98

\begin{tabular}{llll}
\hline Relationship & Correlation & $95 \%$ CI & $\begin{array}{l}\text { Number of } \\
\text { pairs }\end{array}$ \\
\hline Father-mother & 0.020 & $0.016,0.028$ & 67795 \\
Father-child & 0.129 & $0.122,0.136$ & 67795 \\
Father-son & 0.126 & $0.116,0.136$ & 35048 \\
Father-daughter & 0.133 & $0.122,0.144$ & 32747 \\
Mother-child & 0.226 & $0.219,0.231$ & 67795 \\
Mother-son & 0.222 & $0.212,0.232$ & 35048 \\
Mother-daughter & 0.231 & $0.221,0.241$ & 32747 \\
\hline
\end{tabular}

parallel, indicating little or no interaction effects between parental values (that is, the effect of the paternal birth weight is roughly the same within each category of maternal birth weight).

For all the data, the regression of a child's birth weight on the father's birth weight gives a coefficient of 0.137 (SE 0.004), and the regression of a child's birth weight on the mother's birth weight gives a coefficient of 0.252 . When including both parents in the regression the coefficients are slightly lower (0.132 and 0.249), with no significant interaction between the two.

For fathers and mothers who themselves were born with a low birth weight (less than 2500 grams), their birth weight may not always be representative for their genetic potential. When we excluded these fathers and mothers, the above regression coefficients were slightly larger (separate estimates: 0.153 and 0.281 ; and simultaneous estimates 0.148 and 0.278 ). Exactly the same results emerged when we restricted to term born mothers and fathers.

The proportion of offspring with low birth weight was $4.0 \%$ (2702 of 67795$)$. If the mother was above $4000 \mathrm{~g}$ at birth herself, the risk of a low birth weight child was $2.2 \%$ (180 of 8212 ) compared with $9.3 \%$ (209 of 2247) if the mother was below $2500 \mathrm{~g}$ at birth (relative risk 4.2). If the father was above $4000 \mathrm{~g}$ (regardless of maternal birth weight), the risk

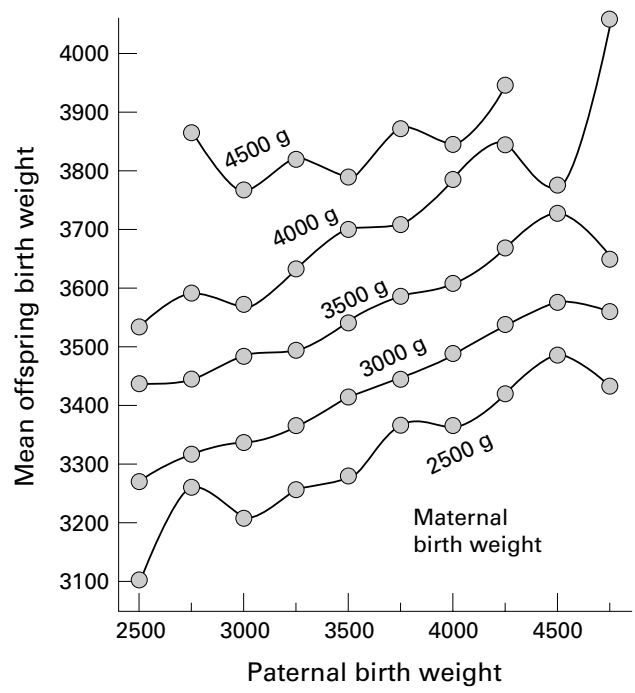

Figure 2 Mean offspring birth weight ( $g$ ) for categories of paternal birth weight in selected strata of maternal birth weight. Families where the parental birth weight was below 2500 grams are omitted from the figure. Maternal and paternal birth weight is categorised into 250 groups - that is, $2500=2500-2749 ; 2750=2750-2999 ; 3000=3000-3249$, etc.
KEY POINTS

- Including paternal birth weight will improve prediction of low birth weight.

- Heritability of birth weight is lower than previous estimates from the same population.

- Social homogamy plays a small part for the population variability in birth weight.

of a low birth weight child was $3.4 \%$ (484 of 14086 ) compared with $6.4 \%$ (112 of 1758) when the father was below 2500 grams (relative risk 1.9). Table 3 shows that the risk is 8.2 times higher when both parents had low birth weight compared with the situation where both parents were above 4000 grams. Within each category of maternal birth weight, the risk of low birth weight in offspring is reduced as the paternal birth weight increases. The table reflects the independent contribution of both parents birth weights to the risk of low birth of the child.

Assuming causality, the proportion of offspring with low birth weight would be reduced from $4.0 \%$ (p) to $3.5 \%$ (q) if parental values were shifted from the two lowest categories to the category with birth weights between 3000 and 3499 grams, using parameters estimated from logistic regression. Thus, the population attributable risk, (p-q)/p, in such an hypothetical situation is 0.125 .

The equations derived from figure 1 are given in table 4 . The spousal correlation was low meaning that $\rho \mathrm{e}^{2}$ must be low, so that the father-child correlation under model 1 will be almost entirely explained by genetic effects. The solution for model 1 is $\mathrm{h}^{2}=0.254(95 \%$ CI: $0.239,0.270), \mathrm{e}^{2}=0.746(0.730,0.761), \rho$ $=0.027(0.018,0.037)$ and $\mathrm{t}_{\mathrm{m}}=0.133(0.120$, $0.146)$.

Table 3 Absolute and relative risk (using the highest parental birth weights as reference) of low birth weight in offspring by combination of maternal and paternal weight categories

\begin{tabular}{|c|c|c|c|c|c|}
\hline \multirow{2}{*}{$\begin{array}{l}\text { Categories of } \\
\text { maternal birth } \\
\text { weight }(g)\end{array}$} & \multirow{2}{*}{$\begin{array}{l}\text { Categories of } \\
\text { paternal birth } \\
\text { weight }(g)\end{array}$} & \multicolumn{2}{|c|}{$\begin{array}{l}\text { Low birth } \\
\text { weight in child }\end{array}$} & \multirow{2}{*}{$\begin{array}{l}\text { Absolute } \\
\text { risk (\%) }\end{array}$} & \multirow{2}{*}{$\begin{array}{l}\text { Relative } \\
\text { risk }\end{array}$} \\
\hline & & Yes & No & & \\
\hline$<2500$ & $<2500$ & 13 & 54 & 19.4 & 8.2 \\
\hline$<2500$ & $2500-2999$ & 18 & 188 & 8.7 & 3.7 \\
\hline$<2500$ & $3000-3499$ & 66 & 654 & 9.2 & 3.9 \\
\hline$<2500$ & $3500-3999$ & 74 & 760 & 8.9 & 3.8 \\
\hline$<2500$ & $4000+$ & 38 & 382 & 9.0 & 3.8 \\
\hline $2500-2999$ & $<2500$ & 26 & 229 & 10.2 & 4.3 \\
\hline 2500-2999 & 2500-2999 & 60 & 856 & 6.6 & 2.8 \\
\hline $2500-2999$ & $3000-3499$ & 208 & 2665 & 7.2 & 3.1 \\
\hline 2500-2999 & $3500-3999$ & 192 & 3286 & 5.5 & 2.3 \\
\hline 2500-2999 & $4000+$ & 90 & 1846 & 4.6 & 2.0 \\
\hline $3000-3499$ & $<2500$ & 40 & 631 & 6.0 & 2.5 \\
\hline $3000-3499$ & 2500-2999 & 132 & 2272 & 5.5 & 2.3 \\
\hline 3000-3499 & $3000-3499$ & 351 & 7435 & 4.5 & 1.9 \\
\hline $3000-3499$ & $3500-3999$ & 375 & 9079 & 4.0 & 1.7 \\
\hline $3000-3499$ & $4000+$ & 193 & 5040 & 3.7 & 1.6 \\
\hline $3500-3999$ & $<2500$ & 26 & 537 & 4.6 & 2.0 \\
\hline 3500-3999 & 2500-2999 & 70 & 1940 & 3.5 & 1.5 \\
\hline 3500-3999 & $3000-3499$ & 214 & 6381 & 3.2 & 1.4 \\
\hline $3500-3999$ & $3500-3999$ & 214 & 8184 & 2.5 & 1.1 \\
\hline $3500-3999$ & $4000+$ & 122 & 4642 & 2.6 & 1.1 \\
\hline $4000+$ & $<2500$ & 7 & 195 & 3.5 & 1.5 \\
\hline $4000+$ & 2500-2999 & 18 & 692 & 2.5 & 1.1 \\
\hline $4000+$ & $3000-3499$ & 56 & 2374 & 2.3 & 1.0 \\
\hline $4000+$ & $3500-3999$ & 58 & 3079 & 1.8 & 0.9 \\
\hline $4000+$ & $4000+$ & 41 & 1692 & 2.4 & 1.0 \\
\hline
\end{tabular}


Table 4 Equations derived from figure 1 for the three phenotypic correlations, assuming either no effect of cultural transmission from the paternal environment (Model 1, $t_{f}=0$ ) or no genetic effects (Model 2, $h=0$ )

\begin{tabular}{lll}
\hline Relationship & Model 1 & Model 2 \\
\hline Mother-father & $\rho \mathrm{e}^{2}$ & $\rho \mathrm{e}^{2}$ \\
Father-child & $1 / 2 \mathrm{~h}^{2}+\rho \mathrm{t}_{\mathrm{m}} \mathrm{e}^{2}$ & $\mathrm{t}_{\mathrm{f}} \mathrm{e}^{2}+\rho \mathrm{t}_{\mathrm{m}} \mathrm{e}^{2}$ \\
Mother-child & $1 / 2 \mathrm{~h}^{2}+\mathrm{t}_{\mathrm{m}} \mathrm{e}^{2}$ & $\rho \mathrm{t}_{\mathrm{e}} \mathrm{e}^{2}+\mathrm{t}_{\mathrm{m}} \mathrm{e}^{2}$ \\
\hline
\end{tabular}

The parameters in model 2 can be uniquely identified using the condition $\mathrm{t}_{\mathrm{f}}{ }^{2}+\mathrm{t}_{\mathrm{m}}{ }^{2}=1$. The estimated parameters are then $\mathrm{e}^{2}=0.244$ (0.236, 0.253), $\rho=0.083(0.053,0.116), \mathrm{t}_{\mathrm{m}}{ }^{2}=$ $0.791(0.762,0.821)$ and $\mathrm{t}_{\mathrm{f}}^{2}=0.209(0.179$, $0.238)$.

\section{Discussion}

The main finding is that paternal birth weight has an independent contribution to offspring birth weight, whether one looks at the whole birth weight distribution or one wants to predict low birth weight in children. The models presented in table 4 explore two possible channels that the parental influence may work through. Assuming no cultural transmission on the father's side $\left(t_{\mathrm{r}}=0\right)$, model 1 demonstrates that the correlations can be explained by a parental genetic effect, leading to an estimated heritability of birth weight of $h^{2}=0.25$. On the other hand, model 2 does not include any genetic effects at all $(\mathrm{h}=0)$, assuming that both maternal and paternal influence is only through cultural transmission, as determined by $t_{m}$ and $t_{f}$. The observed correlations are equally well explained by this somewhat unrealistic scenario. The merit of the model is the division of cultural transmission among the two parents when the generational effect is assumed to be non-genetic. The model predicts that $\mathrm{t}_{\mathrm{f}}^{2}=0.21$, meaning that $21 \%$ of the cultural transmission derives from the paternal side, perhaps a rather high part of the total. The models are both saturated (fit perfectly), and consequently no comparison of goodness of fit is possible. Thus, both models provide possible explanations for the observed data, and it seems reasonable to believe that reality is somewhere in between the two extremes.

In evaluating these results, one should note the selection of subjects to the study population. The first generation consists necessarily of survivors who have given birth to children before the age of 32, while the offspring generation is unselected with respect to future survival and fertility. It is difficult to see what kind of bias this can bring to the correlational structure. Parental age is not known to have large effects on offspring birth weight. However, it seems that there may be a stronger selection to fertility for males than for females in this group of relatively young parents, based on the observation that only $2.6 \%$ of fathers were low birth weight. This observation could be explained by selection of tall men to early fatherhood.

It is relatively well established that maternal birth weight is a good predictor of offspring birth weight. ${ }^{3-6}$ There is less agreement on the interpretation of this observation. It has been argued that pregnancy can have harmful consequences on the later reproductive potential of a female fetus, if it is exposed to adverse environmental factors. ${ }^{11}$ This may be seen as a direct effect, which is not well represented in figure 1, but which would not be possible to distinguish from the cultural transmission on the maternal side in the present dataset. The finding of a substantial correlation between the father and child speaks against this mechanism as a major explanation of the association across generations.

We have not corrected the birth weights for prematurity or for gestational age more generally. For the purpose of predicting the birth weight in the firstborn child, the information on parental birth weights will be useful together with ultrasound measurements during pregnancy, at a time when the length of the pregnancy is yet unknown. For the purpose of understanding the population variability in birth weight, correcting for gestational age may blur the picture, as gestational length is an uncertain measure. Also, gestational age is as much an outcome of pregnancy as birth weight, and it is not obvious which one of these variables may be the cause of the other.

Studies of siblings have shown correlations for birth weight of about $0.5 .^{1}$ Earlier analyses of data from the Medical Birth Registry, using the MZ half-sib method ${ }^{2}$ suggested higher estimates of heritability. Siblings and parentoffspring are expected to have the same degree of correlation under polygenic inheritance. A reasonable interpretation of the higher correlation in sibs is that maternal effects, which may be determined by the maternal genotype (but is the environment seen from the fetal perspective), are responsible for a certain part, maybe $25 \%$, of the population variance in birth weight. This proposition should be tested out in studies of other relationships, in particular half-siblings and cousins.

We assume polygenic inheritance which implies many genes, each with a small effect on the phenotype. As yet, no common allele has been found that has large influences on the variability in birth weight, although some interesting associations of specific genes are emerging. ${ }^{12-13} \mathrm{Simi}-$ larly, there are few examples of common environmental factors that have large effects on birth weight. For instance, in Western societies, maternal nutrition seems to have relatively small influence on birth weight. ${ }^{14}$

The advantage of this study is that the risk of low birth weight can be studied conditional on the birth weight of both parents (table 3). In clinical practice, if low birth weight is to be predicted, the paternal birth weight should be included. It is interesting that paternal birth weight seems to be a better predictor of offspring birth weight than paternal height. ${ }^{15}$ In clinical decisions one should be more cautious in diagnosing, by ultrasound or otherwise, intrauterine growth retardation if both parents were small at birth.

Funding: none.

Conflicts of interest: none.

1 Karn MN, Lang-Brown H, MacKenzie H, et al. Birth weight, gestation time and survival in sibs. Ann Eugen weight, gestation

2 Magnus P. Causes of variation in birth weight. A study of offspring of twins. Clin Genet 1984;25:15-24. 
3 Hackman E, Emanuel I, van Belle G, et al. Maternal birth weight and subsequent pregnancy outcome. $\mathscr{F} A M A 1983$

4 Magnus P, Berg K, Bjerkedal T, et al. Parental determinants of birth weight. Clin Genet 1984;26:397-405.

5 Klebanoff MA, Granbard BI, Kessel SS, et al. Low birth weight across generations. $\mathcal{F} A M A$ 1984;252:2423-7.

6 Emanuel I, Filakti H, Alberman E, et al. Intergenerational studies of human birthweight from the 1958 birth cohort. Brf Obstet Gynaecol 1992;99:836-40.

7 Births in Norway through 30 years. [In Norwegian]. Bergen: Medical Birth Registry of Norway, 1998.

$8 \mathrm{Li}$ CC. Path analysis - a primer. Pacific Grove, CA: The Boxwood Press, 1975

9 Efron B, Tibshirani RJ. An introduction to the bootstrap. New York: Chapman and Hall, 1993.
10 S-PLUS 2000 for Windows. Seattle, WA: MathSoft.

11 Ounsted M, Ounsted C. On fetal growth rate. Clin Dev Med 1973;46:65-72.

12 Hattersley AT, Beards F, Ballantyne E, et al. Mutations in the glucokinase gene of the fetus result in reduced birth weight. Nature Genetics 1998;19:268-70.

13 Dunger DV, Ong KKL, Stewart JH, et al. Association of the INS VNTR with size at birth. Nature Genetics 1998;19:98100.

14 Mathews F, Yudkin P, Neil A. Influence of maternal nutrition on outcome of pregnancy: prospective cohort study. BMF 1999;319:339-43.

15 Wilcox MA, Newton CS, Johnson IR. Paternal influences on birthweight. Acta Obstet Gynecol Scand 1995;74:1518 .

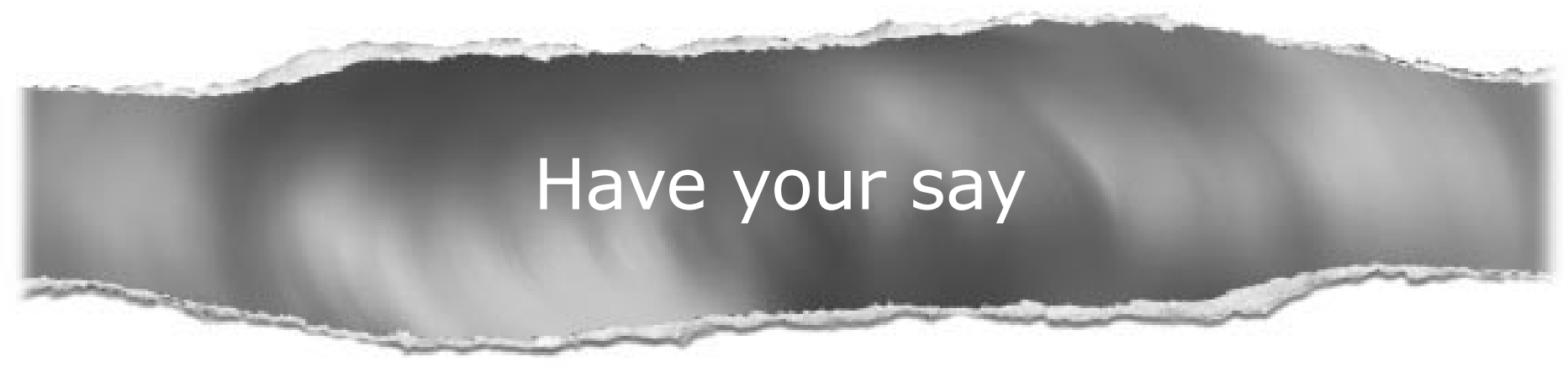

\section{eletters}

If you wish to comment on any article published in the Journal of Epidemiology and Community Health you can send an eLetter using the eLetters link at the beginning of each article. Your response will be posted on Journal of Epidemiology and Community Health online within a few days of receipt (subject to editorial screening).

www.jech.com 